\title{
Size-dependent elasticity of nanocrystalline titania
}

\author{
Bin Chen, ${ }^{1, *}$ Hengzhong Zhang, ${ }^{1}$ K. A. Dunphy-Guzman, ${ }^{1,2}$ D. Spagnoli, ${ }^{1}$ M. B. Kruger, ${ }^{3}$ D. V. S. Muthu,,${ }^{3,4}$ M. Kunz, ${ }^{5}$ \\ Sirine Fakra, ${ }^{5}$ J. Z. Hu, ${ }^{6}$ Q. Z. Guo, ${ }^{6}$ and Jillian F. Banfield ${ }^{1}$ \\ ${ }^{1}$ Earth and Planetary Science, University of California-Berkeley, Berkeley, California 94720, USA \\ ${ }^{2}$ Department of Systems Studies, Sandia National Laboratories, Livermore, California 94551, USA \\ ${ }^{3}$ Department of Physics, University of Missouri, Kansas City, Missouri 64110, USA \\ ${ }^{4}$ Department of Physics, Indian Institute of Science, Bangalore 560012, India \\ ${ }^{5}$ Advanced Light Source, Lawrence Berkeley National Laboratory, Berkeley, California 94720, USA \\ ${ }^{6}$ National Synchrotron Light Source, Brookhaven National Laboratory, Upton, New York 11973, USA \\ (Received 19 December 2008; revised manuscript received 23 January 2009; published 4 March 2009)
}

\begin{abstract}
Synchrotron-based high-pressure x-ray diffraction measurements indicate that compressibility, a fundamental materials property, can have a size-specific minimum value. The bulk modulus of nanocrystalline titania has a maximum at particle size of $15 \mathrm{~nm}$. This can be explained by dislocation behavior because very high dislocation contents can be achieved when shear stress induced within nanoparticles counters the repulsion between dislocations. As particle size decreases, compression increasingly generates dislocation networks (hardened by overlap of strain fields) that shield intervening regions from external pressure. However, when particles become too small to sustain high dislocation concentrations, elastic stiffening declines. The compressibility has a minimum at intermediate sizes.
\end{abstract}

DOI: $10.1103 /$ PhysRevB.79.125406

PACS number(s): 62.25.-g, 61.05.cp, 61.46.Hk, 81.07.Wx

\section{INTRODUCTION}

Nanoscale physics has attracted considerable experimental and theoretical interest. Size effects on the plasticity of materials have been widely investigated. ${ }^{1-3}$ The increase in hardness and yield strength of materials with decreasing grain size, known as the Hall-Petch effect, has been attributed to increase in dislocation pileups in small particles. ${ }^{4,5}$ Deviation from the Hall-Petch effect has been reported in particles in nanometer size range. ${ }^{6}$ Several mechanisms are proposed for the inverse Hall-Petch effect, ${ }^{7-11}$ such as the mechanisms based on dislocations, ${ }^{12}$ diffusion, ${ }^{13,14}$ grainboundary shearing, ${ }^{15}$ core-shell structures ${ }^{16,17}$ two-phase structures, ${ }^{18}$ collective motion, ${ }^{19}$ energy gain and cohesive energy loss, ${ }^{20}$ and dislocation-absorption models. ${ }^{21}$ In contrast to size effects on plasticity, the size dependence of elasticity is poorly understood. ${ }^{22-28}$ Using synchrotron-based $\mathrm{x}$-ray diffraction (XRD) we measured on nanocrystalline titania $\left(\mathrm{TiO}_{2}\right)$ sized in a wide range. Here we report a finding: that the compressibility of nanomaterials has a minimum value at a specific particle size $\left(15 \mathrm{~nm}\right.$ for $\mathrm{TiO}_{2}$ nanoparticles). We also propose the mechanisms that most likely explain the observations. Our work focuses on titania because it is a well-studied model material of wide technological relevance. Titania is widely used in ceramics, catalysis, electronics, and metallurgy. The changes in particle size may change the material properties or processing performance. A good understanding of the size effect of its elasticity is important to its application, as well as the scientific exploration of nanophysics.

\section{EXPERIMENT}

Samples with anatase structure and particle diameters of $4.0,6.5,7.2,9.0,13.5,15.0,21.3,24.0,30.1$, and $45.0 \mathrm{~nm}$ were prepared by heating amorphous titania in air for spe- cific lengths of time. ${ }^{29}$ Small nanocrystalline $(<5 \mathrm{~nm})$ anatase samples were prepared using the method of Scolan and Sanchez. ${ }^{30}$ Because anatase is metastable with respect to rutile at larger particle sizes and it transforms to rutile during the coarsening, ${ }^{31-33}$ pure anatase samples with particle sizes $>6 \mathrm{~nm}$ were prepared from solid amorphous titania. Amorphous titania was synthesized by controlled hydrolysis of titanium ethoxide and heated in air at temperatures from 375 to $550{ }^{\circ} \mathrm{C}$ for $3 \mathrm{~h}$ to produce single-phase anatase powders with discrete average particle sizes ranging from 7 to $50 \mathrm{~nm}$. The phase composition and the average particle size of each sample were analyzed by XRD. The transmission electron microscope (TEM) and the Brunauer-Emmett-Teller (BET) were also used to confirm the size determination based on XRD. Details have been reported previously. ${ }^{29}$

A spring-steel or rhenium gasket, with a chamber having a diameter of $130 \mu \mathrm{m}$, was used to contain the sample between diamonds with $350 \mu \mathrm{m}$ culets (Fig. 1). ${ }^{34}$ A 4:1 mixture of methanol:ethanol was used as a pressure-transmitting medium in order to maintain a quasihydrostatic environment. In addition, a small amount of ruby $(<2 \%)$ was included for pressure determination from its fluorescence shifts. Synchrotron $\mathrm{x}$-ray diffraction patterns were collected at pressure intervals of $1 \sim 2 \mathrm{GPa}$ (Fig. 2). At beamline 11.3.1 of ALS, Lawrence Berkeley National Laboratory, angular dispersive XRD is measured. The wavelength is $0.7327 \AA$. The maximum diffraction angle is near $45^{\circ}$. At beamline X17C of NSLS, Brookhaven National Laboratory, energy-dispersive $\mathrm{XRD}$ is measured. The energy of the light is up to $\sim 80 \mathrm{keV}$. The diffraction angle is set to $\sim 10^{\circ}$. The diffraction pattern is collected about $20 \sim 30 \mathrm{~min}$ for each pressure. Using Rietveld structural refinement [Fig. 3(a)] we determined the lattice parameter of titania at each pressure (Table I) and analyzed the data in terms of the Birch-Murnaghan equation of state [Table II; Fig. 3(b), Ref. 36] 


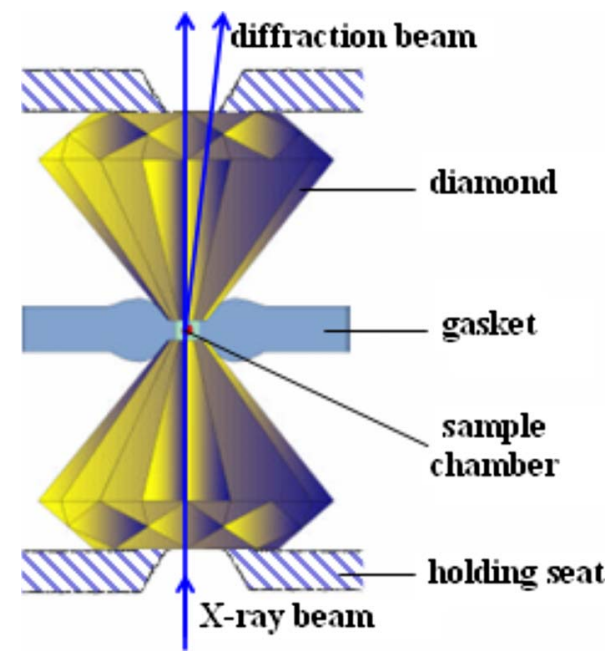

FIG. 1. (Color online) Schematic diagram of the high-pressure $\mathrm{x}$-ray diffraction experimental setup. Two well-aligned diamonds, pressing against a sample contained in a gasket hole, constitute the core of a diamond anvil cell (DAC). Diamond culets are very small (usually several hundred microns across), and a gentle force can generate a very high pressure. Diamonds are very hard and can stand higher pressure than all other known materials. The good transparency of diamonds to radiation including visible light and x-rays allows optical and spectroscopic measurements on pressurized samples. Diamond anvil cell techniques are well established for bulk modulus measurements and have been widely used for several decades despite that the realization of perfectly hydrostatic compression with DACs is still challenging (image courtesy of Serge Desgreniers).

$$
P=1.5 K\left[\left(\frac{V}{V_{0}}\right)^{-7 / 3}-\left(\frac{V}{V_{0}}\right)^{-5 / 3}\right]\left\{1-\frac{3}{4}\left(4-K^{\prime}\right)\left[\left(\frac{V}{V_{0}}\right)^{-2 / 3}-1\right]\right\},
$$

where $V / V_{0}$ is the ratio of unit cell volume at pressure $P$ and ambient pressure. $K$ is the bulk modulus, and $K^{\prime}$ is the pressure derivative of bulk modulus.

\section{COMPUTATIONAL MODELING}

Bulk anatase and anatase nanoparticles (1.6, 2.5, 3.0, and $3.6 \mathrm{~nm}$ in diameter) were run in molecular dynamics (MD)

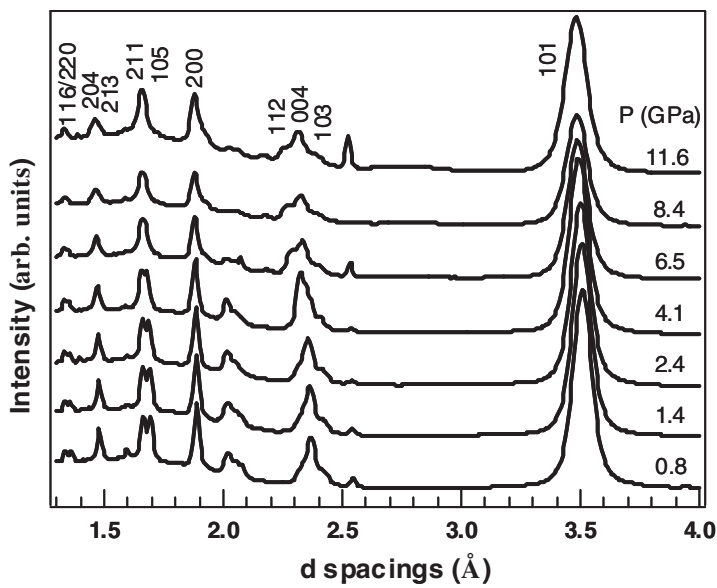

FIG. 2. Representative XRD patterns of $24 \mathrm{~nm} \mathrm{TiO}_{2}$ particles in compression. The unlabeled lines are from the gasket.
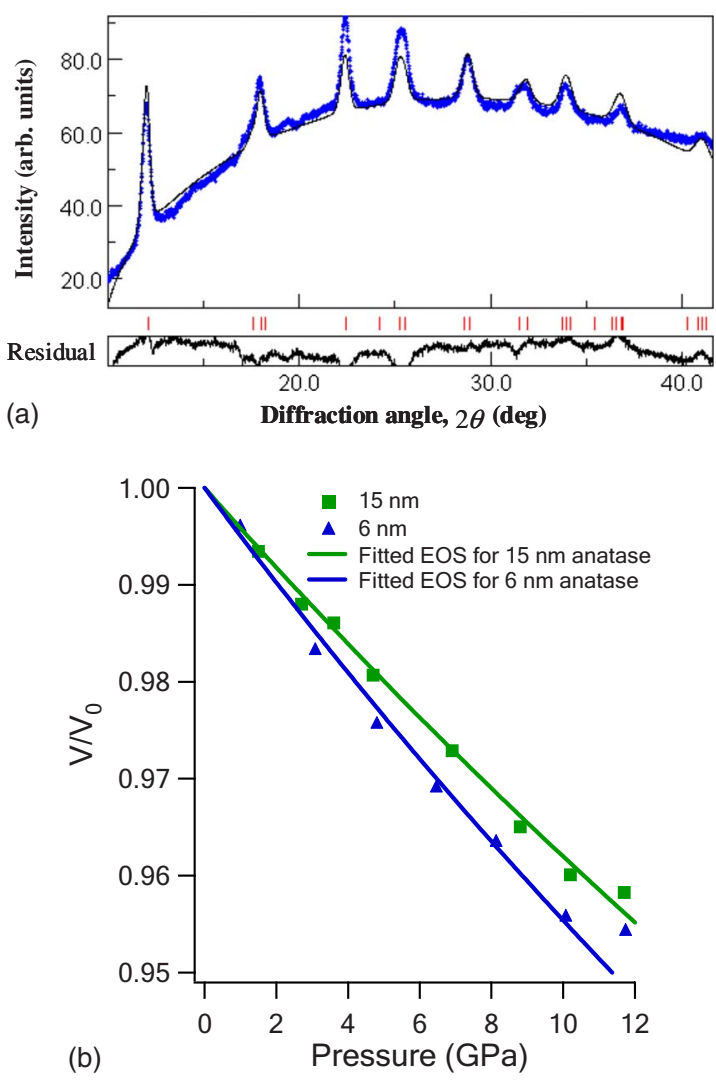

FIG. 3. (Color online) (a) Representative Rietveld structural refinement fit of $15 \mathrm{~nm} \mathrm{TiO} 2$ particles. The broad bump background is due to diffuse scattering. (b) Representative unit cell volume ratio and fitted equation of state.

simulations under increasing pressure. The simulations are based on the Born model of solids, ${ }^{38}$ which is comprised of two parts. The long-range interactions are described by Coulombic forces, and the short-range interactions between ions are modeled by simple parameterized analytical functions, which aim to represent repulsion between electron-charge clouds and van der Waals attraction forces. The potentials used to describe the interactions between titanium and oxygen in anatase are those of Matsui and Akaogi. ${ }^{39}$ A noninteracting medium was used to apply external pressure to the nanoparticles. The anatase nanoparticles were incased in argon, where the argon potential is described by a simple Lennard-Jones interaction. ${ }^{40}$ As the argon atoms are included in the simulation to pressurize the nanoparticles, the interactions of the Ti-Ar and $\mathrm{O}-\mathrm{Ar}$ are represented by their closest closed shell species; i.e., the Ne-Ar Lennard-Jones potential is used for the O-Ar interaction. All the anatase nanoparticle structures were generated using the METADISE code. ${ }^{41}$ The molecular dynamics simulations were performed using DL_POLY 2.14. ${ }^{42}$ The starting configurations of all simulations were relaxed at $300 \mathrm{~K}$, and no applied pressure for a MD time of $1 \mathrm{~ns}$ worth of data. The end configuration from these relaxed structures was then used as the starting point for the high-pressure study. The argon atoms were then used to incase the nanoparticles, and pressure was increased from 1 to $12 \mathrm{GPa}$. During the course of the simulations the pres- 
TABLE I. Diffraction lines of pressurized anatase of $15 \mathrm{~nm}$. The values are reported in angstrom.

\begin{tabular}{|c|c|c|c|c|c|c|c|c|c|c|c|c|}
\hline $\begin{array}{l}P \\
(\mathrm{GPa})\end{array}$ & $d 101$ & $d 004$ & $d 200$ & $d 105$ & $d 211$ & $d 204$ & $d 116$ & $d 220$ & $d 215$ & $d 224$ & $a$ & $c$ \\
\hline Bulk $^{\mathrm{a}}$ & 3.5169 & 2.3785 & 1.8925 & 1.7001 & 1.6665 & 1.4809 & 1.3642 & 1.3382 & 1.2647 & 1.1663 & 3.785 & 9.514 \\
\hline$A m b^{b}$ & 3.5110 & 2.3652 & 1.8905 & 1.6921 & 1.6645 & 1.4767 & 1.3582 & 1.3368 & 1.2608 & 1.1638 & 3.781 & 9.461 \\
\hline 0.6 & 3.5106 & 2.3652 & 1.8903 & 1.6921 & 1.6643 & 1.4766 & 1.3582 & 1.3366 & 1.2608 & 1.1637 & 3.781 & 9.461 \\
\hline 1.5 & 3.5064 & 2.3497 & 1.8896 & 1.6831 & 1.6634 & 1.4725 & 1.3514 & 1.3362 & 1.2568 & 1.1615 & 3.779 & 9.399 \\
\hline 2.7 & 3.5018 & 2.3412 & 1.8878 & 1.6779 & 1.6617 & 1.4696 & 1.3475 & 1.3349 & 1.2541 & 1.1597 & 3.776 & 9.365 \\
\hline 3.6 & 3.4998 & 2.3389 & 1.8869 & 1.6764 & 1.6609 & 1.4686 & 1.3463 & 1.3343 & 1.2532 & 1.1589 & 3.774 & 9.356 \\
\hline 4.7 & 3.4941 & 2.3331 & 1.8841 & 1.6726 & 1.6584 & 1.4658 & 1.3433 & 1.3323 & 1.2508 & 1.1569 & 3.768 & 9.332 \\
\hline 6.9 & 3.4864 & 2.3233 & 1.8806 & 1.6663 & 1.6551 & 1.4617 & 1.3384 & 1.3298 & 1.2472 & 1.1541 & 3.761 & 9.293 \\
\hline 8.8 & 3.4788 & 2.3128 & 1.8772 & 1.6596 & 1.6520 & 1.4575 & 1.3333 & 1.3274 & 1.2434 & 1.1512 & 3.754 & 9.251 \\
\hline 10.2 & 3.4739 & 2.3064 & 1.8750 & 1.6556 & 1.6500 & 1.4549 & 1.3302 & 1.3258 & 1.2410 & 1.1494 & 3.750 & 9.226 \\
\hline 11.7 & 3.4735 & 2.3009 & 1.8754 & 1.6525 & 1.6503 & 1.4537 & 1.3279 & 1.3261 & 1.2398 & 1.1490 & 3.751 & 9.204 \\
\hline
\end{tabular}

The lattice parameters are from Ref. 35 from which the $d$ spacing is calculated.

${ }^{\mathrm{b}}$ Ambient pressure.

sure was increased at $1 \mathrm{GPa}$ increments at $500 \mathrm{ps}$ intervals for the 1.6 and $2.5 \mathrm{~nm}$ nanoparticles and $1 \mathrm{~ns}$ intervals for the 3.0 and $3.6 \mathrm{~nm}$ nanoparticles. All simulations used a NPT ensemble (constant number of ions, pressure, and temperature). At $300 \mathrm{~K}$ and during the equilibration period of $100 \mathrm{ps,}$ the temperature and pressure were controlled by a Berendsen thermostat and barostat, with a relaxation time of 1 ps.

\section{RESULTS AND DISCUSSION}

\section{A. Observed size-dependent compressibility}

To avoid complications due to the onset of a phase transformation at higher pressure, only the XRD data for pressures below $12 \mathrm{GPa}$ were used for the calculation of the bulk modulus. XRD peak narrowing did not occur during compression (Fig. 2), suggesting no significant pressure-induced particle coarsening. In fact, peak broadening increases during compression due to increasing strain inhomogeneity and microstrains (Fig. 1). Differences in the elastic properties of nanoparticle interiors and their grain-boundary regions, particle aggregation, and increased nonhydrostaticity due to unavoidable solidification of liquid pressure media likely account for local strain inhomogeneity. Development of some preferred orientation changed the relative intensities of diffraction peaks, but the peak positions used to determine lattice parameters, which contain the information of macrostrain in the elastic deformation, are not affected.
Hooke's Law, $\varepsilon=S \sigma$, describes the elastic properties of a material, where $S$ is the compliance constant from which elastic moduli including Young's Modulus, shear modulus, and bulk modulus can be calculated. ${ }^{43}$ Bulk modulus (the inverse of compressibility) describes how the volume of a material changes during elastic deformation. It can be determined from changes in the lattice parameters of titania at each pressure. Lattice parameter information extracted from experimental data (Table I) was analyzed in terms of the Birch-Murnaghan equation of state (Fig. 3). ${ }^{36}$ It is found that the bulk modulus increases as the particle size decreases to $15 \mathrm{~nm}$ in diameter and then decreases in particles less than $15 \mathrm{~nm}$ in diameter (Fig. 4; Table II). The current study shows that the bulk modulus, as hardness, has a maximum at a specific size and suggests that the mechanisms of plastic and elastic deformation in nanoparticles may be related.

\section{B. Reduced compressibility due to increased dislocation contents in stressed nanocrystals}

The size dependence of hardness has been attributed to dislocations, which can either strengthen or soften materials depending on their mobility. ${ }^{44}$ It is widely accepted that in particles of micron size range, dislocation pileups increase as particle size decreases, leading to greater dislocation densities because pileups impede removal of dislocations. ${ }^{4,5}$ However, the role of dislocations in nanoparticles is considered limited because repulsive interactions between them result in

TABLE II. Bulk modulus of anatase with a series of particle sizes. Values with asterisks are from our MD simulations. The measured bulk modulus of bulk anatase is from Ref. 37. The others are from the measurements of this study. $15 \mathrm{~nm} \mathrm{TiO}_{2}$ nanoparticles were measured twice.

\begin{tabular}{|c|c|c|c|c|c|c|c|c|c|c|c|c|c|c|c|}
\hline $\begin{array}{l}\text { Size } \\
(\mathrm{nm})\end{array}$ & 3.0 & 3.6 & 4.0 & 6.5 & 7.2 & 9.0 & 13.5 & 15.0 & 15.0 & 21.3 & 24.0 & 30.1 & 45.0 & bulk & bulk \\
\hline$K(\mathrm{GPa})$ & $131^{*}$ & $143^{*}$ & 185 & 199 & 211 & 230 & 237 & 239 & 245 & 234 & 228 & 226 & 215 & 179 & $167^{*}$ \\
\hline$\Delta K(\mathrm{GPa})$ & & & 2.6 & 1.5 & 5.0 & 5.1 & 1.5 & 4.8 & 4.2 & 2.6 & 6.3 & 4.6 & 5.7 & 2.0 & \\
\hline
\end{tabular}




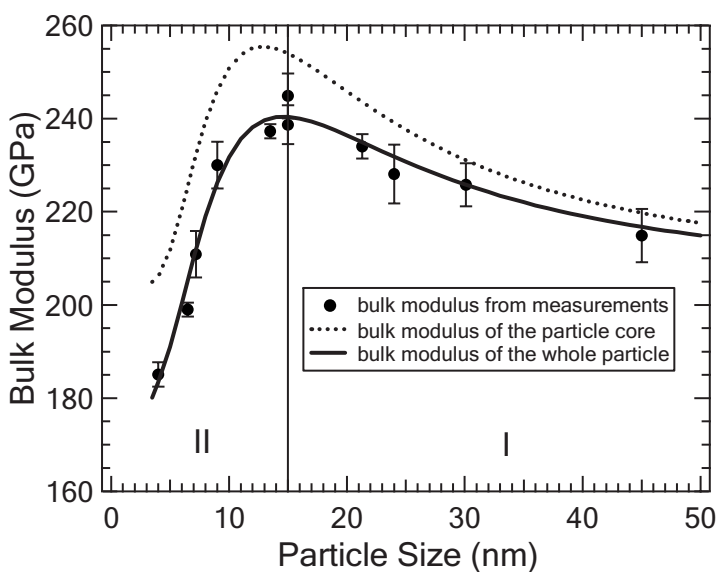

FIG. 4. Size dependence of the bulk modulus of titania nanoparticles. Two sets of high-pressure XRD measurements are made for $15 \mathrm{~nm} \mathrm{TiO}_{2}$ particles. The solid curve represents the effective bulk modulus of the whole particle (core and shell) that is fitted with the model described by Eqs. (2) and (3). The dashed curve is the fitted bulk modulus of the particle core.

low numbers of dislocations per particle..$^{45,46}$ Although so, increased numbers of dislocations have been observed in stressed nanomaterials $\mathrm{s}^{47}$ because shear stress can decrease the equilibrium distances between dislocations and between dislocations and the surface (Fig. 5). The force on per unit length of two edge dislocations separated by a distance of $l_{d d}$ is $f=G b^{2} / 2 \pi(1-\nu) l_{d d}{ }^{48}$ In the magnitude estimation of the interaction force, published values of $90 \mathrm{GPa},{ }^{49} 0.27,{ }^{49}$ and $0.4 \mathrm{~nm}(\operatorname{Ref} 46)$ are used for shear modulus $(G)$, Poisson's ratio $(\nu)$, and Burgers vector $(b)$ of titania, respectively. The image force on per unit length of an edge dislocation at a distance of $l_{d s}$ from the particle surface is $f=G b^{2} / 4 \pi(1$ $-\nu) l_{d s}{ }^{50}$ The applied stress needed for canceling out dislocation interaction force or image force is $\sigma_{\text {ext }}=f / b .{ }^{48} \mathrm{Al}$ though more complicated dislocations may exist in real cases, the simplified calculation above is useful for rough estimation of dislocation concentration in compression. At room pressure, the predicted equilibrium spacing of disloca-

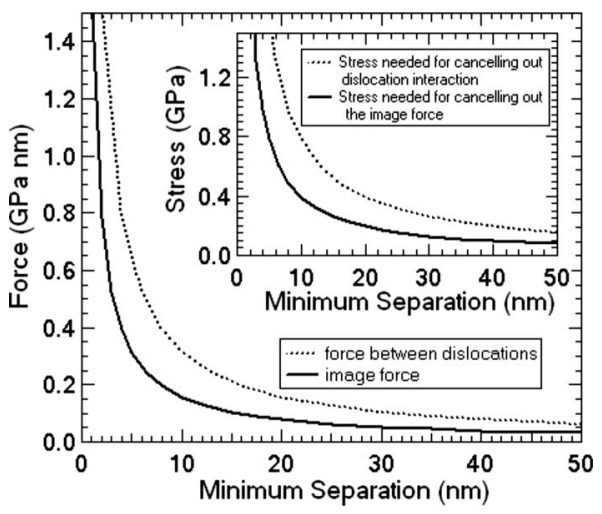

FIG. 5. Diagram showing the force between dislocations and between surface and dislocation as a function of their separation. Inset shows the external stress needed to achieve this range of dislocation separations.

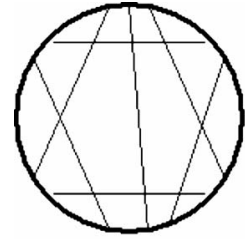

(a)

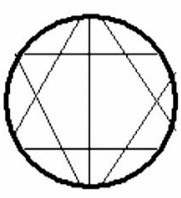

(b)

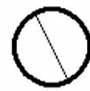

(c)
FIG. 6. Illustration of dislocation networks. Circles represent particles of materials, and lines represent dislocations.

tions in titania is on the order of $100 \mathrm{~nm}$. An external shear stress of 1.3 GPa could overcome repulsion and could allow two dislocations to move within $6 \mathrm{~nm}$ of each other, so long as dislocation motion is not impeded by other factors (Fig. 5).

Ashby and Johnson ${ }^{51}$ observed that hydrostatic pressure might induce shear stress and dislocation formation if crystals contain regions of different elastic properties. This is likely the case for nanoparticles due to different compressibility of the core and surface regions. Our measurements of compressibility involve external pressures of up to $12 \mathrm{GPa}$. Based on the fitted results of our measured data, the bulk moduli of the grain boundaries and particle cores are $\sim 139$ and 205-258 GPa, respectively. Using the formula of Ashby and Johnson, ${ }^{51}$ we estimate the maximum shear stress in the sample to be $11.8 \%-18.5 \%$ of the applied pressure. These values actually are underestimated because (1) the sample particles are not perfectly spherical; (2) the applied pressure is not ideally hydrostatic. Based on the calculations above, we predict that GPa levels of shear stress can be induced within nanoparticles. Consequently, significant numbers of dislocations are predicted for nanoparticles a few tens of nanometer in diameter and larger.

In stressed nanomaterials, dislocation pileups will become more prevalent as particle size decreases due to increasing interaction of dislocations with interfaces and each other. Dislocation networks within materials under high pressure are strained regions that resist compression more than intervening regions because the atoms are already displaced from their equilibrium positions. Networks of dislocations thus can shield intervening regions (from which the majority of the XRD signal originates) from external pressure (Fig. 6), reducing the compressibility with decrease in particle size. We propose that the enhanced overlap of dislocation strain fields in smaller particles accounts for their reduced compressibility. Evidence that dislocations can influence elastic properties can be inferred from previous reports of increasing elastic moduli with increasing strain of bulk materials. ${ }^{52,53}$ Consequently, we infer that dislocations could contribute to elastic stiffening in microsized and tens of nanometer-scale particles (range I in Fig. 4).

\section{Enhanced compressibility of very fine nanocrystals due to the dominant contribution of surface layers}

A notable feature of Fig. 4 is the decrease in bulk modulus with decrease in particle size for particles less than $15 \mathrm{~nm}$ in 
diameter. In particles of all sizes, dislocations that are very close to nanoparticle surfaces are likely to be unstable because the defects can be removed readily by diffusion. Thus, the trend toward more dislocations with reducing size must reverse below some critical particle diameter. When the crystallite size is comparable to the equilibrium distance between dislocations (range II in Fig. 4), networks that are sufficiently large to shield intervening regions cannot form. We infer that loss of pressure shielding becomes more pronounced as the particle size of titania decreases below $15 \mathrm{~nm}$. It is not possible to visualize or quantify dislocation concentrations during compression. Elimination of dislocations during decompression is likely, ${ }^{47}$ so post compression characterization of dislocation concentrations cannot provide direct confirmation of dislocation concentrations at high pressure, making it necessary to rely on predictions for this analysis.

Dislocation mechanisms cease in very small particles. The contribution of the surface layer dominates the elastic behavior more in smaller nanoparticles. In situ high-pressure Mössbauer spectroscopy has been used to demonstrate the reduced atomic density and enhanced compressibility of the surface layers of nanocrystalline iron. ${ }^{54}$ Surface layers have open and less rigid structures and thus have smaller shear modulus and bulk modulus than interiors. We propose that the absence of interlocking dislocation networks and the increased contribution of more compressible surface layers in very small particles explain why the bulk modulus decreases with decreasing particle size.

\section{THEORETICAL MODELS}

\section{A. Model for the size dependence of compressibility}

It is normally believed that the surface regions of nanoparticles differ from regions in nanoparticle interiors due to strain arising from the presence of atoms with incomplete coordination shells. Based on this construct, we develop a model to describe the response of nanoparticles to compression. For simplicity, the nanoparticles may be viewed to be composed of a spherical crystalline interior surrounded by a disordered layer at the grain boundary. The measured bulk modulus is the effective bulk modulus that reflects the contributions of the bulk modulus of the grain interior $\left(K_{i}\right)$ and bulk modulus of outer interface layer $\left(K_{o}\right) .{ }^{55}$ The bulk modulus of any amorphous layer (beyond the disordered layer) cannot be analyzed by XRD. If such regions exist, they can be viewed as part of pressure media. The composite spheres model $^{56}$ gives the effective bulk modulus, $K$, as

$$
K=K_{o}+\frac{c\left(K_{i}-K_{o}\right)}{1+(1-c) \frac{K_{i}-K_{o}}{K_{o}+\frac{4}{3} G_{o}}},
$$

where $G_{o}$ is the shear modulus of the boundary and $c$ is the volume fraction of the particle interior.

Anatase is tetragonal. However, because the experiments involve primarily randomly oriented particles, isotropic structure is approximated for simplicity. (Notes: preferred orientation introduces an orientation dependence of shear modulus and Poisson's ratio. This will influence the numeri- cal values of coefficients that relate the bulk modulus to the shear modulus. However, the related coefficients are determined by fitting in this analysis. The proposed mechanism will not be affected.) Consequently, the bulk modulus of the particle interior can be written as $K_{i}=\frac{2\left(1+v_{i}\right)}{1-2 v_{i}} G_{i}$, where $v_{i}$ is Poisson's ratio. The ratio of shear modulus and hardness is denoted as $G_{i} / \tau_{i}=H_{i}$, and we assume that the particle core follows the Hall-Petch-type relation, $\tau_{i}=\tau_{0}+c_{\tau}\left(d-2 t_{g b}\right)^{-n / 2}$, where $d$ is the particle size, $t_{g b}$ the thickness of the disorder surface layer, $\tau_{0}, c_{\tau}$ and $n$ are coefficients. Below a particle size of a certain value $\left(d_{c}\right)$, dislocation pileups decrease and then disappear-ultimately eliminating dislocation strengthening. To reflect this behavior, we modify the Hall-Petch relation to a more general form, $\tau_{i}=\tau_{0}+c_{\tau}\left(\frac{d}{d_{c}}\right) d_{c} / d(d$ $\left.-2 t_{g b}\right)^{-n / 2}$. Accordingly, for the bulk modulus of the particle core,

$$
K_{i}=K_{i}^{*}+c_{k}\left(\frac{d}{d_{c}}\right)^{d_{c} / d}\left(d-2 t_{g b}\right)^{-n / 2},
$$

where $K_{i}^{*}$ and $c_{k}$ are coefficients.

Similarly for boundary layers, the bulk modulus can be written as $K_{o}=\frac{2\left(1+v_{o}\right)}{1-2 v_{o}} H_{o} \tau_{o}$, where $v_{o}$ is Poisson's ratio and $H_{o}$ denotes $G_{o} / \tau_{o}$ (shear modulus/hardness). As discussed above, boundary deformation mechanisms often involve several processes. As a generalized description, the hardness can be written as $\tau_{o}=\tau_{o}^{*}+\sum_{m=-\infty}^{\infty} c_{m} t_{g b}^{-m / 2}$, where $\tau_{0}^{*}$ and $c_{m}$ are coefficients. We assume that the surface layer thickness does not vary dramatically with changing particle $\operatorname{size}^{26}$ and treat the boundary hardness and bulk modulus as particle-sizeindependent quantities.

Fitting the measured bulk moduli for particles of different sizes with the model [Eqs. (2) and (3)], we estimate the thickness of the surface layer as $\sim 0.2 \mathrm{~nm}$ and its bulk modulus as $\sim 139 \mathrm{GPa}$. As shown in Fig. 4, surface softening, even confined in a very limited region, could significantly affect the effective elasticity of the whole particle. The fitting generates the critical particle size $d_{c}=15.8 \mathrm{~nm}$. This value is in good agreement with the apparent position of the maximum of the effective bulk modulus at $\sim 15 \mathrm{~nm}$. The dependence of bulk modulus on $d^{-3 / 2}$ is generated in our fit, in contrast to the classical dependence of hardness on $d^{-1 / 2}$.

\section{B. Compressibility enhancement of very fine nanocrytals confirmed with molecular dynamics simulations}

A series of molecular dynamics simulations were performed for four different-sized anatase nanoparticles (1.6, $2.5,3.0$, and $3.6 \mathrm{~nm}$ in diameter) and bulk anatase to further examine the size dependence of compressibility. MD simulations for $10 \mathrm{~nm}$ and larger nanoparticles are beyond the present computationally accessible range. However, simulations for smaller particles may provide some insights into nanoparticle response to pressure. The predicted bulk moduli are 167,143 , and $131 \mathrm{GPa}$ for bulk and 3.6- and 3-nmdiameter $\mathrm{TiO}_{2}$ particles, respectively, reasonably close to the values measured experimentally. For 1.6 and $2.5 \mathrm{~nm}$ particles, the structures are quite amorphous, as shown in the simulated XRD pattern (Fig. 7) and we got no meaningful 


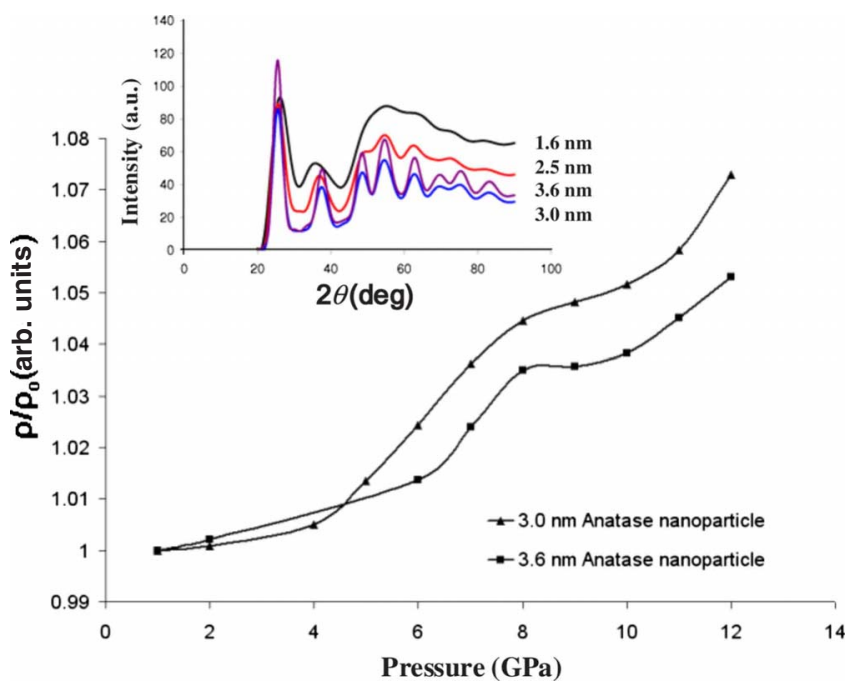

FIG. 7. (Color online): Simulated density ratio as a function of pressure for the 3.0 and $3.6 \mathrm{~nm}$ sized nanoparticles. Inset: simulated XRD patterns based on MD simulations of $\mathrm{TiO}_{2}$ nanoparticles.

bulk modulus. However the simulated XRD pattern of 3.0 and $3.6 \mathrm{~nm}$ shows a more crystalline structure. By calculating the change in density as a function of pressure we observe that after $5 \mathrm{GPa}$ the $3.0 \mathrm{~nm}$ nanoparticle is more compressible than the $3.6 \mathrm{~nm}$ nanoparticle (Fig. 7), which is consistent with the experimentally observed trend.

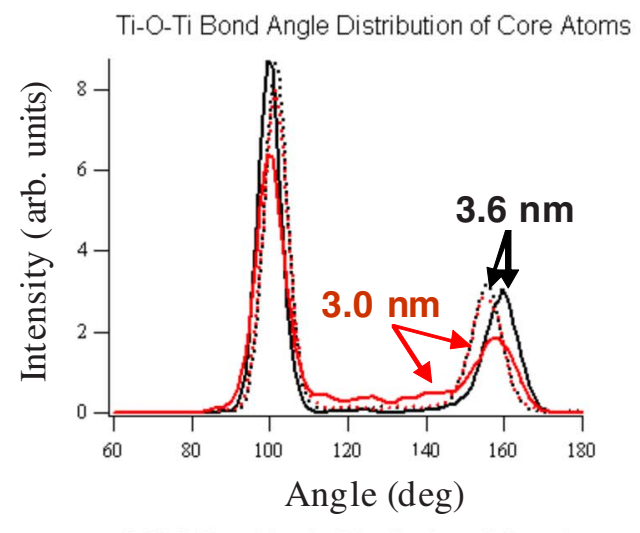

O-Ti-O Bond Angle Distribution of Core Atoms

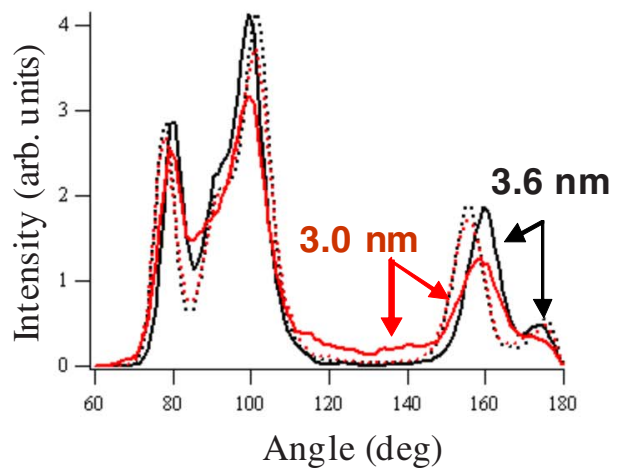

To investigate further the reasoning behind the difference in compressibility of the $3.0 \mathrm{~nm}$ nanoparticle compared to the $3.6 \mathrm{~nm}$ nanoparticle, we analyzed the effects on the surface atoms and core atoms as pressure is increased. We can separate the surface atoms from the core atoms in the MD simulation and analyze the change in bond angle distribution of the O-Ti-O and the Ti-O-Ti bond angles. The bond angle distribution of the surface O-Ti-O atoms and the Ti-O-Ti atoms in the $3.0 \mathrm{~nm}$ nanoparticle at low pressure shows much less structure than the $3.6 \mathrm{~nm}$ nanoparticle (Fig. 8). The peaks are much sharper and larger in the $3.6 \mathrm{~nm}$ low pressure simulation compared to the $3.0 \mathrm{~nm}$ nanoparticle at the same pressure. As the surface structure is less ordered, in the $3.0 \mathrm{~nm}$ nanoparticle, the change in bond angle distribution when pressure is increased is greater in these core atoms compared with the core atoms in the $3.6 \mathrm{~nm}$ nanoparticle. This could give reasons behind the increased compressibility of the $3.0 \mathrm{~nm}$ nanoparticle compared with the $3.6 \mathrm{~nm}$ nanoparticle. Our simulations support the inference from fitting experimental data that very small $\mathrm{TiO}_{2}$ nanoparticles $(3.6 \mathrm{~nm}$ in diameter or smaller) are more compressible than bulk material.

\section{CONCLUSION}

We have shown that the compressibility of nanosized $\mathrm{TiO}_{2}$ has a minimum value at a specific particle size. We

Ti-O-Ti Bond Angle Distribution of Surface Atoms

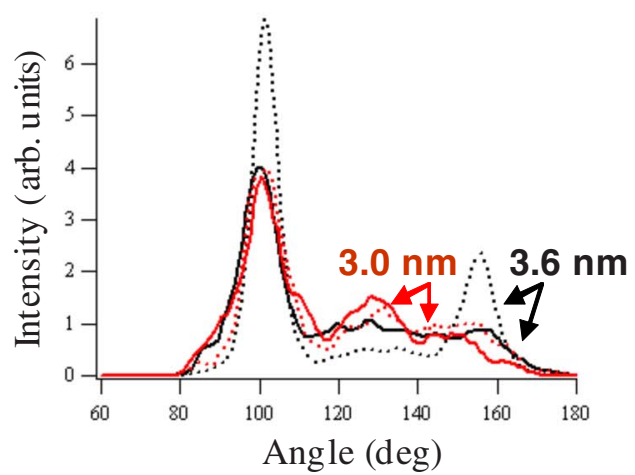

O-Ti-O Bond Angle Distribution of Surface Atoms

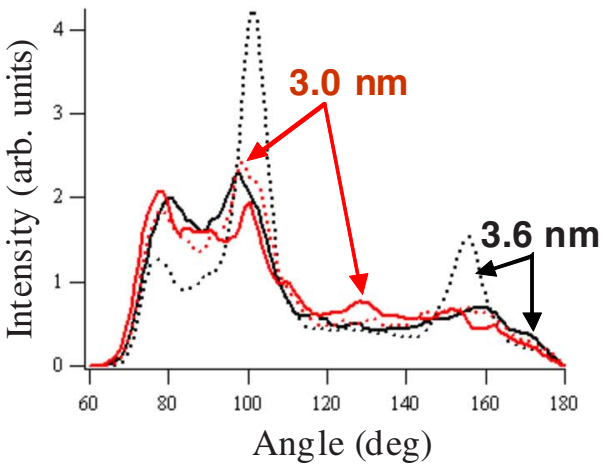

FIG. 8. (Color online) Bond angle distribution plots of the core and surface atoms from the MD simulation of the 3.0 (red or as labeled) and 3.6 (black or as labeled) nm nanoparticles at 1 (dashed) and 12 (solid) GPa. The core bond angles change more in the $3.0 \mathrm{~nm}$ nanoparticle compared with the $3.6 \mathrm{~nm}$ nanoparticle. The surface structure of the $3.0 \mathrm{~nm}$ nanoparticle is less ordered than the surface structure of the $3.6 \mathrm{~nm}$ nanoparticle. 
think that elastic stiffening conferred by dislocation networks increases as size decreases until particles become too small to sustain high dislocation concentrations, leading to a sizespecific compressibility minimum. This work should be of broad interest because elastic properties are an intrinsic characteristic of all solids. Understanding their correlation with nanoscale defects will help understand properties of nanomaterials. The results may be found useful in materials technology for optimizing the mechanical performance of nanomaterials to meet specific needs of applications.

\section{ACKNOWLEDGMENTS}

High-pressure x-ray diffraction was performed at beamline 11.3.1 of Advanced Light Source (ALS), Lawrence Ber- keley National Laboratory and beamlines X17C \& X17B3 of the National Synchrotron Light Source (NSLS), Brookhaven. We thank J. Giska, M. Finnegan, F. El-Ghussein, and T. Tesileanu for help with the synchrotron measurements; Sergio Speziale and Raymond Jeanloz for their DAC cells; Benjamin Gilbert for helpful discussion; Stephen C. Parker for providing us with the computer code METADISE and for the useful discussions. The authors thank Glenn A. Waychunas for the provision of the Geochemistry computer cluster at the Lawrence Berkeley National Laboratory. Research conducted at the ALS is supported by the Office of Science, Basic Energy Sciences, Division of Materials Science of the U.S. Department of Energy under Contract Nos. DE-AC0205CH11231 and DE-AC02-05CH11232. Financial support for this work was provided by the U.S. Department of Energy (Grant No. DE-FG03-01ER15218).
*Author to whom correspondence should be addressed; binchen@berkeley.edu

${ }^{1}$ Zhiwei Shan, E. A. Stach, J. M. K. Wiezorek, J. A. Knapp, D. M. Follstaedt, and S. X. Mao, Science 305, 654 (2004).

${ }^{2}$ M. Chen, E. Ma, K. J. Hemker, Hongwei Sheng, Yinmin Wang, and Xuemei Cheng, Science 300, 1275 (2003).

${ }^{3}$ S. X. McFadden, R. S. Mishra, R. Z. Valiev, A. P. Zhilyaev, and A. K. Mukherjee, Nature (London) 398, 684 (1999).

${ }^{4}$ E. O. Hall, Proc. Phys. Soc. London, Sect. B 64, 747 (1951).

${ }^{5}$ N. J. Petch, J. Iron Steel Inst. 174, 25 (1953).

${ }^{6}$ A. H. Chokshi, A. Rosen, J. Karch, and H. Gleiter, Scr. Metall. 23, 1679 (1989).

${ }^{7}$ J. Schiøtz and K. W. Jacobsen, Science 301, 1357 (2003).

${ }^{8}$ J. Schiøtz, F. D. Di Tolla, and K. W. Jacobsen, Nature (London) 391, 561 (1998).

${ }^{9}$ S. Yip, Nature (London) 391, 532 (1998).

${ }^{10}$ C. C. Koch and J. Narayan, in Structure and Mechanical Properties of Nanophase Materials-Theory and Computer Simulations vs. Experiment, edited by D. Farkas et al., MRS Symposia Proceedings No. 634 (Materials Research Society, Warrendale, PA, 2001), p. B5.1.1.

${ }^{11}$ V. Yamakov, D. Wolf, S. R. Phillpot, A. K. Mukherjee, and H. Gleiter, Philos. Mag. Lett. 83, 385 (2003).

${ }^{12}$ J. C. Li, Trans. Soc. Pet. Eng. AIME 227, 239 (1963).

${ }^{13}$ Y. Mishin, D. Farkas, M. J. Mehl, and D. A. Papaconstantopoulos, Phys. Rev. B 59, 3393 (1999).

${ }^{14}$ H. P. Van Swygenhoven, P. M. Derlet, and A. G. Frøseth, Nature Mater. 3, 399 (2004)

${ }^{15}$ F. A. Mohamed, Metal. Mater. Trans. 38, 340 (2007).

${ }^{16}$ H. Bei, S. Xie, and E. P. George, Phys. Rev. Lett. 96, 105503 (2006).

${ }^{17}$ B. Palosz, S. Stel'makh1, E. Grzanka, S. Gierlotka, R. Pielaszek, U. Bismayer, S. Werner, and W. Palosz, J. Phys.: Condens. Matter 16, S353 (2004).

${ }^{18}$ G. J. Fan, H. Choo, P. K. Liaw, and E. J. Lavernia, Mater. Sci. Eng., A 409, 243 (2005).

${ }^{19}$ F. Louchet, J. Weiss, and T. Richeton, Phys. Rev. Lett. 97, 075504 (2006).

${ }^{20}$ P. Yong, Y. Zhou, and C. Sun, SciencePaper Online. See http:// www.paper.edu.cn/en/paper.php?serial_number=200710-109.
${ }^{21}$ C. E. Carlton and P. J. Ferreira, Acta Mater. 55, 3749 (2007).

${ }^{22}$ B. Chen, D. Penwell, L. R. Benedetti, R. Jeanloz, and M. B. Kruger, Phys. Rev. B 66, 144101 (2002).

${ }^{23}$ B. Chen, D. Penwell, M. B. Kruger, A. F. Yue, and B. Fultz, J. Appl. Phys. 89, 4794 (2001).

${ }^{24}$ B. Chen, D. Penwell, and M. B. Kruger, Solid State Commun. 115, 191 (2000)

${ }^{25}$ J. Zhang, Y. Zhao, and B. Palosz, Appl. Phys. Lett. 90, 043112 (2007).

${ }^{26}$ M. D. Kluge, D. Wolf, J. F. Lutsko, and S. R. Phillpot, J. Appl. Phys. 67, 2370 (1990).

${ }^{27}$ B. Gilbert, H. Zhang, B. Chen, M. Kunz, F. Huang, and J. F. Banfield, Phys. Rev. B 74, 115405 (2006).

${ }^{28}$ S. M. Clark, S. G. Prilliman, C. K. Erdonmez, and A. P. Alivisatos, Nanotechnology 16, 2813 (2005).

${ }^{29}$ H. Z. Zhang, M. Finnegan, and J. F. Banfield, Nano Lett. 1, 81 (2001).

${ }^{30}$ E. Scolan and C. Sanchez, Chem. Mater. 10, 3217 (1998).

${ }^{31}$ X. Liu, H. Wang, D. Chen, Y. Wang, L. Lu, and X. Wang, J. Appl. Polym. Sci. 73, 2569 (1999).

${ }^{32}$ W. F. Zhang, M. S. Zhang, and Z. Yin, Phys. Status Solidi A 179, 319 (2000)

${ }^{33}$ H. Zhang and J. F. Banfield, J. Mater. Chem. 8, 2073 (1998).

${ }^{34}$ H. K. Mao, P. M. Bell, K. J. Dunn, R. M. Chrenko, and R. C. DeVries, Rev. Sci. Instrum. 50, 1002 (1979).

${ }^{35}$ G. V. Samsonov, The Oxide Handbook (IFI/Plenum, New York, 1982).

${ }^{36}$ F. Birch, J. Geophys. Res. 83, 1257 (1978).

${ }^{37}$ T. Arlt, M. Bermejo, M. A. Blanco, L. Gerward, J. Z. Jiang, J. S. Olsen, and J. M. Recio, Phys. Rev. B 61, 14414 (2000).

${ }^{38}$ M. Born and K. Huang, Dynamical Theory of Crystal Lattices, 1st ed. (Oxford University Press, Oxford, 1954).

${ }^{39}$ M. Matsui and M. Akaogi, Mol. Simul. 6, 239 (1991).

${ }^{40}$ M. L. Klein and J. A. Venables, Rare Gas Solids (Academic, London, 1977), Vol. 2.

${ }^{41}$ G. W. Watson, E. T. Kelsey, N. H. de Leeuw, D. J. Harris, and S. C. Parker, J. Chem. Soc., Faraday Trans. 92, 433 (1996).

${ }^{42}$ W. Smith and T. R. Forester, J. Mol. Graph. 14, 136 (1996).

${ }^{43}$ J. F. Nye, Physical Properties of Crystals (Oxford University 
Press, London, U.K., 1992).

${ }^{44}$ A. Lasalmonie and J. L. Strudel, J. Mater. Sci. 21, 1837 (1986).

${ }^{45}$ V. G. Gryaznov, I. A. Polonsky, A. E. Romanov, and L. I. Trusov, Phys. Rev. B 44, 42 (1991).

${ }^{46}$ R. L. Penn and J. F. Banfield, Science 281, 969 (1998).

${ }^{47}$ Z. W. Shan, J. M. K. Wiezorek, E. A. Stach, D. M. Follstaedt, J. A. Knapp, and S. X. Mao, Phys. Rev. Lett. 98, 095502 (2007).

${ }^{48}$ T. G. Nieh and J. Wadsworth, Scr. Metall. Mater. 25, 955 (1991).

${ }^{49}$ The "AZo Journal of Materials Online," CERAM Research Ltd. See http://www.azom.com/details.asp?ArticleID=1179.

${ }^{50}$ J. P. Hirth and J. Lothe, Theory of Dislocations, 1st ed. (Wiley,
New York, 1982), p. 88.

${ }^{51}$ M. F. Ashby, L Johnson, Phil. Mag. 19, 757 (1969); 20, 1009 (1969).

${ }^{52}$ W. Yang, T. Tsakalakos, and J. E. Hilliard, J. Appl. Phys. 48, 876 (1977).

${ }^{53}$ A. F. Jankowski and T. Tsakalakos, J. Phys. F: Met. Phys. 15, 1279 (1985).

${ }^{54}$ S. Trapp, C. T. Limbach, U. Gonser, S. J. Campbell, and H. Gleiter, Phys. Rev. Lett. 75, 3760 (1995).

${ }^{55}$ R. M. Christensen, J. Elast. 50, 15 (1998).

${ }^{56}$ Z. Hashin, J. Appl. Mech. 29, 143 (1962). 